\title{
Root Submergence Technique: Forgotten Terrain Revisited
}

\author{
Dr. Rejina Shrestha, ${ }^{1}$ Dr. Shaili Pradhan, ${ }^{1}$ Dr. Ranjita Shrestha Gorkhali, ${ }^{1}$ Dr. Anand Verma ${ }^{2}$ \\ ${ }^{1}$ Periodontology \& Oral Implantology Unit, Department of Dental Surgery, National Academy of Medical Sciences, Bir \\ Hospital, Kathmandu, Nepal; 'Prosthodontics Unit, Department of Dental Surgery, National Academy of Medical Sciences, \\ Bir Hospital, Kathmandu, Nepal
}

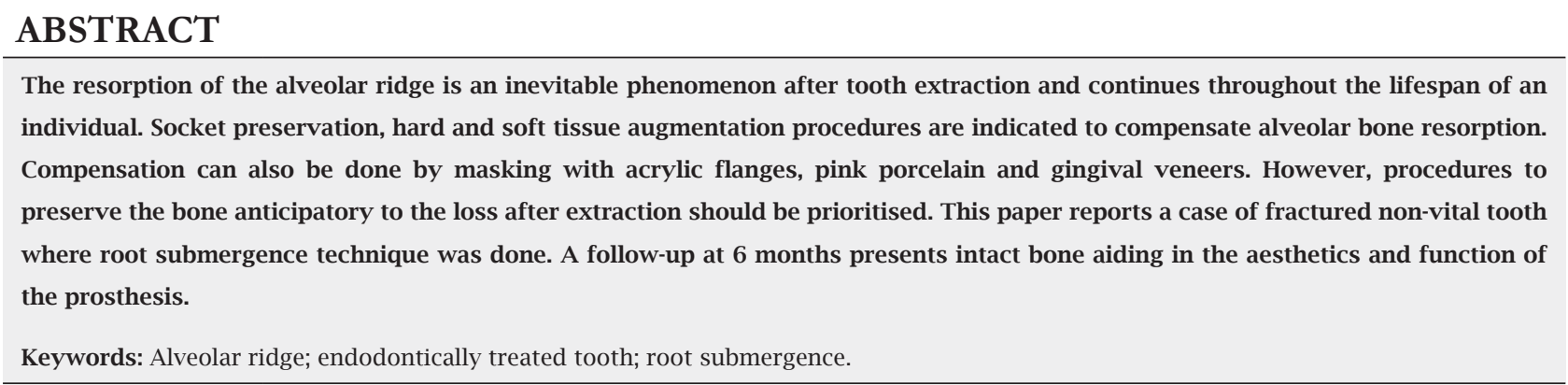

\section{INTRODUCTION}

Partial extraction therapies (PET) are a subgroup of therapies that use the tooth itself to compensate the loss of alveolar tissue and prevent the collapse of the ridge. ${ }^{1}$ They include root submergence, socket shield, pontic shield and proximal socket shield techniques.

The root submergence technique or the root banking² was introduced in the 1950s in prosthodontics to maintain the alveolar ridge under complete dentures. The technique involves complete removal of the coronal portion of the tooth leaving behind an intact root.

The advantage of this technique is that it preserves bone width, bone height, the connective tissue and the blood supply of the indicated tooth which ultimately helps to maintain papilla height and width and eliminates the need for extensive bone and connective tissue grafting surgery. ${ }^{3}$ Moreover, the clinician will still have an option of socket shield technique before implant placement for future purpose.

The indications ${ }^{4}$ are unrestorable tooth crown with absence of apical pathology. The tooth should have healthy

\footnotetext{
Correspondence:

Dr. Rejina Shrestha

Periodontology and Oral Implantology Unit, Dental Department, National Academy of Medical Sciences, Bir Hospital, Kathmandu, Nepal.

email: rejinashrestha811@gmail.com

Citation

Shrestha R, Pradhan S, Gorkhali RS, Verma A. Root submergence Technique: Forgotten Terrain Revisited. J Nepal Soc Perio Oral Implantol. 2018;2(2):73-5.
}

amputated pulp or endodontic therapy must have been completed. However, there are certain contraindications to the procedure which includes apical pathologies, root caries, root resorption, ankylosis, periapical pathology, endo-perio lesion and soft tissue perforation. ${ }^{3}$

\section{CASE REPORT}

A 62-year-old male patient reported to the Periodontology and Oral Implantology Unit with the chief complaint of broken upper front teeth since two weeks. His medical history revealed diabetes mellitus, hypertension and hypothyroidism. Intraoral examination revealed fractured tooth in relation to 23 and 33, generalised attrition, generalised abrasion, missing 17, 16, 15, 12, 22, 25, 26, 27, 37,47 , crown in relation to 11 with defective margin and bridge in relation to $34,35,36$.

Various treatment options were consulted with the patient. The patient agreed to comprehensive restoration with root submergence technique followed by removable partial denture. The treatment plan included oral prophylaxis, restorations and replacement of faulty prosthesis. The tooth had been endodontically treated previously and showed no periapical pathology on the periapical radiograph.

Procedure: Scaling and root planing was done followed by oral hygiene instructions. Following phase I therapy, the root submergence technique was performed. Patient preparation was done (Figure 1 a) with proper extraoral asepsis with $2 \%$ povidone-iodine, followed by intraoral preprocedural $0.2 \%$ chlorhexidine rinses. Local anaesthesia was administered.

Crevicular incision was given with Bard Parker blade no. 12. Vertical incisions was given with Bard Parker blade no. 15. 

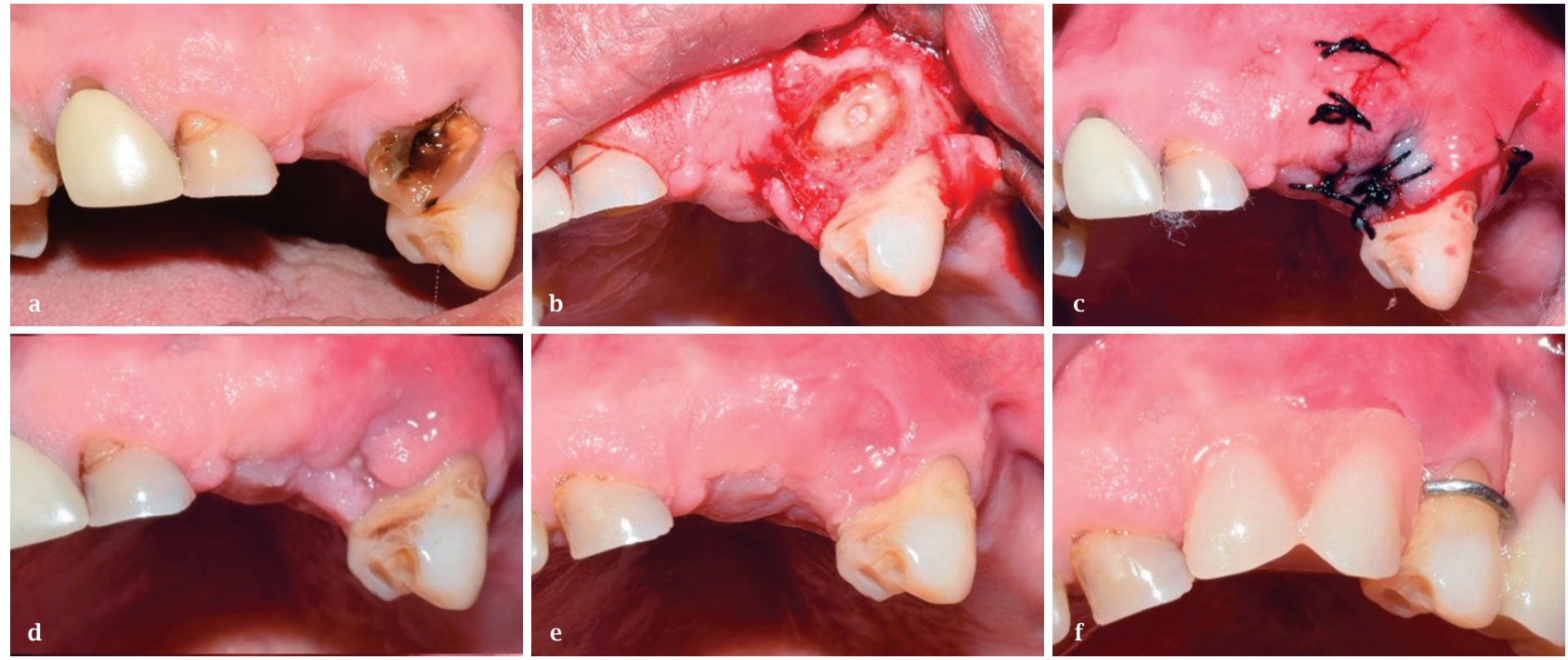

Figure 1: (a) On clinical Examination (b) Decoronation (c) Suture placed (d) Suture removal (e) Healed site (f) Prosthesis.
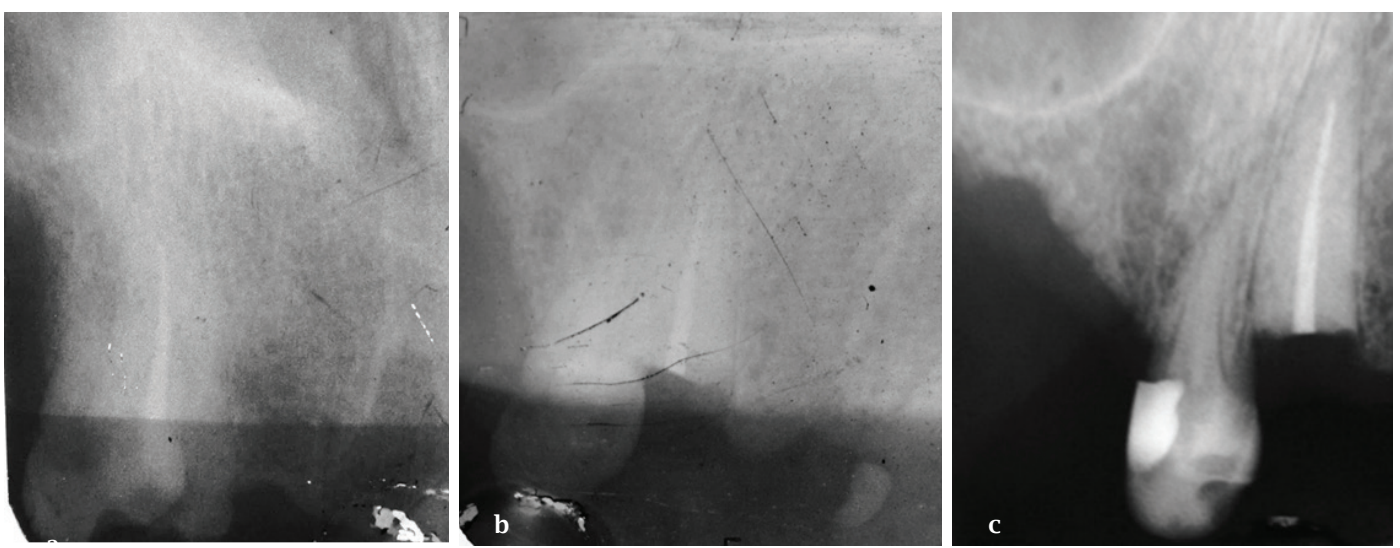

Figure 2: Intraoral Periapical Radiograph (a) Initial (b) Immediately after surgery (c) After six months.

Labial and lingual mucoperiosteal flaps were raised for the advancement and mobility of the flap. The root was reduced to $2 \mathrm{~mm}$ below the level of the alveolar crest using high speed handpiece with straight bur (Figure $1 \mathrm{~b}$ ). Interrupted suturing technique with 3-0 silk was performed (Figure $1 \mathrm{c}$ ). The patient was prescribed analgesics (Ibuprofen $400 \mathrm{mg}$ every 8 hours) or when needed and instructed to rinse with $0.2 \%$ every 12 hours for 14 days. Postoperative written instructions were given. Sutures were removed after seven days (Figure $1 \mathrm{~d}$ ). There was uneventful healing (Figure 1 e). Removable partial denture made up of acrylic and acrylic teeth with clasp was delivered after three months (Figure $1 \mathrm{f}$ ).

Intra oral periapical radiograph at six months revealed intact alveolar bone with no pathologies (Figure 2 a-c). The patient was satisfied with the treatment and the same procedure was done to rehabilitate the left lower canine.

\section{DISCUSSION}

Hard and soft tissue augmentations before, during, or after implant placement have shown successful results. But, they require surgical expertise, longer duration time, sound health and financial condition of the patient. Immediate implant placement is another option to minimise bone loss at the extraction site, but its success is limited by the tissue biotype and buccal bone thickness. Socket preservation minimises the amount of resorption but does not preserve the alveolar ridge dimensions and the height of the papilla completely. ${ }^{5}$

Partial extraction therapy can be opted as a potential treatment modality for the preservation of the bone when other modalities are restricted due to systemic diseases and financial constraints.

The first reported clinical study of submerged endodontically treated roots in humans was done by Howell in 1977.2 He recommended that the procedure could be utilised for the maintenance of ridge height under prosthetic appliances.

The root submergence technique is possible in both vital and non-vital roots. In non-vital root submergence, the tooth is endodontically treated before decoronation.

In vital root submergence, the vital tooth is decoronated, keeping the pulp intact and ensuring that the root is covered 
with a flap. The pulp tissue in the roots remains vital because of the blood supply through the apices and collateral occlusal circulation from the soft tissue. ${ }^{6}$ Whitaker and Shankle in 1974 showed that vitally submerged teeth have a greater repair potential compared with endodontically treated teeth. ${ }^{7}$ The criteria ${ }^{8}$ for vital root submergence include one $\mathrm{mm}$ horizontal mobility, reducible infrabony defects, one third root length/ bone ratio, asymptomatic vital teeth and healthy mucogingival tissue.

The complications of the root submergence technique are failure of the submerged roots that could result in their extraction, subsequent grafting of the site, and rehabilitation with a new prosthesis. ${ }^{4,9}$ The complication in this case would be soft tissue perforation. Von Wowern and Winther ${ }^{9}$ reported a four-year Follow-up study of 20 non-vital submerged roots among 15 participants. The study revealed 53\% failures due to exposure of the root surface. In order to prevent this complication, the root should be completely submerged, and all sharp edges should be removed.

In a study done by $\mathrm{O}^{\prime} \mathrm{Neal},{ }^{10}$ histologic and radiographic findings showed positive results and they concluded that root submergence technique should be considered as an alternative to extraction of key teeth in an effort to preserve alveolar bone.

Although current reports support the success of the technique, the procedure has not been indicated as a routine procedure due to lack of long term evidence. Thus, clinical cases should be studied in detail and proper case selection is the key to the success of this therapy.

\section{REFERENCES}

1. Gluckman H, Salama M, Du Toit J Partial Extraction Therapies (PET) Part 1: Maintaining Alveolar Ridge Contour at Pontic and Immediate Implant Sites. Int J Periodontics Restorative Dent. 2016;36(5):681-7.

2. Dugan DJ, Getz JB, Epker BN. Root banking to preserve alveolar bone: a review and clinical recommendation. J Am Dent Assoc. 1981;103(5):737-43.

3. Çomut A, Mehra M, Saito H. Pontic site development with a root submergence technique for a screw-retained prosthesis in the anterior maxilla. J Prosthet Dent. 2013;110(5):337-43.

4. Salama M, Ishikawa T, Salama H, Funato A, Garber D. Advantages of the root submergence technique for pontic site development in esthetic implant therapy. Int J Periodontics Restorative Dent. 2007;27(6):521-7.

5. Nevins M, Camelo M, De Paoli S, Friedland B, Schenk RK, Parma-Benfenati S. A study of the fate of the buccal wall of extraction sockets of teeth with prominent roots. Int J Periodontics Restorative Dent. 2006;26(1):19-29.

6. Guyer SE. Selectively retained vital roots for partial support of overdentures: a patient report. J Prosthet Dent. 1975;33(3):258-63.

7. Whitaker DD, Shankle RJ. A study of the histologic reaction of submerged root segments. Oral Surg Oral Med Oral Pathol. 1974;37(6):919-35.

8. Garver DG, Fenster RK. Vital root retention in humans: a final re $\neg$ port. J Prosthet Dent. 1980;43(4):368-73.

9. Von Wowern N, Winther S. Submergence of roots for alveolar ridge preservation. A failure (4-year follow-up study). Int J Oral Surg. 1981;10:247-50.

10. O'Neal RB, Gound T, Levin MP, del Rio CE. Submergence of roots for alveolar bone preservation I. Endodontically treated roots. Oral Surg Oral Med Oral Pathol. 1978;45(5):803-10. 